\title{
Improved Generic Attacks against Hash-Based MACs and HAIFA*
}

\author{
Itai Dinur ${ }^{1}$ and Gaëtan Leurent ${ }^{2}$ \\ 1 Département d'Informatique, École Normale Supérieure, Paris, France \\ Itai.Dinur@ens.fr \\ 2 Inria, EPI SECRET, France \\ Gaetan.Leurent@inria.fr
}

\begin{abstract}
The security of HMAC (and more general hash-based MACs) against state-recovery and universal forgery attacks was very recently shown to be suboptimal, following a series of surprising results by Leurent et al. and Peyrin et al.. These results have shown that such powerful attacks require much less than $2^{\ell}$ computations, contradicting the common belief (where $\ell$ denotes the internal state size). In this work, we revisit and extend these results, with a focus on properties of concrete hash functions such as a limited message length, and special iteration modes.

We begin by devising the first state-recovery attack on HMAC with a HAIFA hash function (using a block counter in every compression function call), with complexity $2^{4 \ell / 5}$. Then, we describe improved trade-offs between the message length and the complexity of a state-recovery attack on HMAC. Consequently, we obtain improved attacks on several HMAC constructions used in practice, in which the hash functions limit the maximal message length (e.g., SHA-1 and SHA-2). Finally, we present the first universal forgery attacks, which can be applied with short message queries to the MAC oracle. In particular, we devise the first universal forgery attacks applicable to SHA-1 and SHA-2.
\end{abstract}

Keywords: Hash functions, MAC, HMAC, Merkle-Damgård, HAIFA, state-recovery attack, universal forgery attack, GOST, Streebog, SHA family.

\section{Introduction}

MAC algorithms are an important symmetric cryptography primitive, used to verify the integrity and authenticity of messages. First, the sender appends to the message a tag, computed from the message and a key. The receiver can recompute the tag using the key and reject the message when the computed tag does not match the received one. The main security requirement of a MAC is the resistance to existential forgery. Namely, after querying the MAC oracle to obtain the tags of some carefully chosen messages, it should be hard to forge a valid tag for a different message.

\footnotetext{
* Some of the work presented in this paper was done during Dagstuhl Seminar 14021.
} 
One of the most widely used MAC algorithms in practice is HMAC, a MAC construction using a hash function designed by Bellare, Canetti and Krawczyk in 1996 [4. The algorithm has been standardized by ANSI, IETF, ISO and NIST, and is widely deployed to secure internet communications (e.g. SSL, SSH, IPSec). As these protocols are widely used, the security of HMAC has been extensively studied, and several security proofs 34 show that it gives a secure MAC and a secure PRF up to the birthday bound (assuming good properties of the underlying compression function). At the same time, there is a simple existential forgery attack on any iterative MAC with an $\ell$-bit state, with complexity $2^{\ell / 2}$, matching the security proof. Nevertheless, security beyond the birthday bound for stronger attacks (such as state-recovery and universal forgery) is still an important topic.

Surprisingly, the security of HMAC beyond the birthday bound has not been thoroughly studied until 2012, when Peyrin and Sasaki described an attack on HMAC in the related-key setting [19. Later work focused on single-key security, and included a paper by Naito, Sasaki, Wang and Yasuda [17, which described state-recovery attacks with complexity $2^{\ell} / \ell$. At Asiacrypt 2013, Leurent, Peyrin and Wang [16] gave state-recovery attacks with complexity $2^{\ell / 2}$, closing the gap with the security proof. More recently, at Eurocrypt 2014, Peyrin and Wang [20] described a universal forgery attack with complexity as low as $2^{5 \ell / 6}$. The complexity of the universal forgery attack was further improved to $2^{3 \ell / 4}$ in [9], showing that even this very strong attack is possible with less than $2^{\ell}$ work.

These generic attacks have also been used as a first step to build specific attacks against HMAC with the concrete hash function Whirlpool [1110].

These very recent and surprising results show that more work is needed to better understand the exact security provided by HMAC and hash-based MACs.

\subsection{Our Results}

In this paper, we provide several important contributions to the security analysis of HMAC and similar hash-based MAC constructions. In particular, we devise improved attacks when HMAC is used with many popular concrete hash functions, and in several cases our attacks are the first to be applicable to HMAC with the given hash function. Some results with concrete instantiations are summarized in Table 1]

As a first contribution, we focus on the HAIFA [5] mode of operation, used in many recent designs such as BLAKE [12], Skein [8], or Streebog [7]. The HAIFA construction uses a block counter to tweak the compression functions, such that they resemble independent random functions, in order to thwart some narrowpipe attacks (e.g. the second-preimage attack of Kelsey and Schneier [14). Indeed, the recent attacks against HMAC [16 20] use in a very strong way the assumption that the same compression function is applied to all the message blocks, and thus they cannot be applied to HAIFA. In this work, we present the first state-recovery attack on HMAC using these hash functions, whose optimal complexity is $2^{4 \ell / 5}$. 
Merkle-Damgård mode

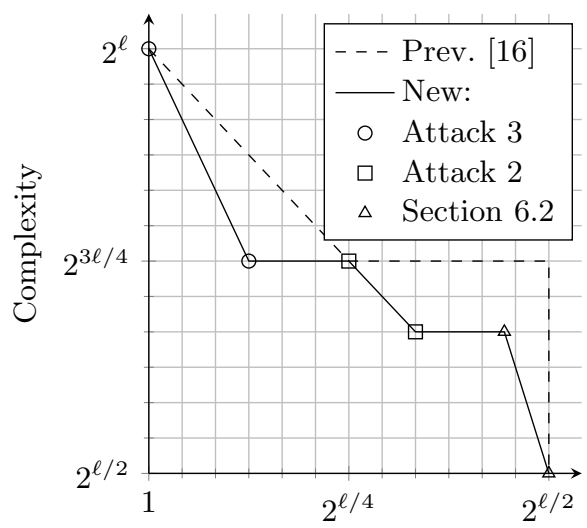

Length of the messages
HAIFA mode

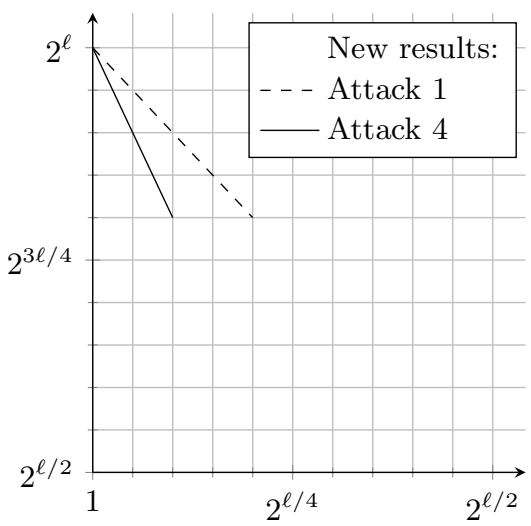

Length of the messages

Fig. 1. Trade-offs between the message length and the complexity

In an interesting application of our state-recovery attack on HAIFA (given in the full version of this paper [6]), we show how to extend it into a key-recovery attack on the new Russian standard Streebog, recovering the 512-bit key of HMAC-Streebog with a complexity of $2^{410}$. This key recovery attack is similar to the one of [16] for Merkle-Damgård, and confirms its surprising observation: adding an internal checksums in a hash function (such as Streebog) weakens the design when used in HMAC, even for hash functions based on the HAIFA mode.

As a second contribution of this paper, we revisit the results of [16], and give a formal proof of the conjectures used in its short message attacks. Some of our proofs are of broad interest, as they give insight into the behavior of classical collision search algorithms for random functions. These proofs explain for the first time an interesting phenomenon experimentally observed in several previous works (such as [18]), namely, that the collisions found by such algorithms are likely to belong to a restricted set of a surprisingly small size.

Then, based on our proofs, we describe several new algorithms with various improved trade-offs between the message length and the complexity as shown in Figure 1. As many concrete hash functions restrict the message size, we obtain improved attacks in many cases: for instance, we reduce the complexity of a state-recovery attack against HMAC-SHA-1 from $2^{120}$ to $2^{107}$ (see Table 1).

Finally, we focus on universal forgery attacks, and devise attacks using techniques which are different from those of [20. While the attack of [20] (and its improvement in [9]) is much more efficient than exhaustive search, it requires, in an inherent way, querying the MAC oracle with very long messages of about $2^{\ell / 2}$ blocks, and thus has limited impact in practice. On the other hand, our attacks can be efficiently applied with much shorter queries to the MAC oracle, and thus have many more applications. In particular, we devise the first universal forgery attack applicable to HMAC with SHA-1 and SHA-2 (see Table 1). 
Table 1. Complexity of attacks on HMAC instantiated with some concrete hash functions. The state size is denoted as $\ell$, and the maximum message length as $2^{s}$. For the new results, we give a reference to the Attack number, or the full version of the paper.

\begin{tabular}{|c|c|c|c|c|c|c|c|}
\hline \multirow[b]{2}{*}{ Function } & \multirow[b]{2}{*}{ Mode } & \multirow[b]{2}{*}{$\ell$} & \multirow[b]{2}{*}{$s$} & \multicolumn{2}{|c|}{ State-recovery } & \multicolumn{2}{|c|}{ Universal forgery } \\
\hline & & & & 16 & New & 20 & New \\
\hline SHA-1 & $\mathrm{MD}$ & 160 & $2^{55}$ & $2^{120}$ & $2^{107}$ & $\mathrm{~N} / \mathrm{A}$ & $2^{132}$ \\
\hline SHA-256 & $\mathrm{MD}$ & 256 & $2^{55}$ & $2^{201}$ & $2^{152}$ & $\mathrm{~N} / \mathrm{A}$ & $2^{228}$ \\
\hline SHA -512 & $\mathrm{MD}$ & 512 & $2^{118}$ & $2^{394}$ & $2^{282}$ & $\mathrm{~N} / \mathrm{A}$ & $2^{453}$ \\
\hline HAVAL & $\mathrm{MD}$ & 256 & $2^{54}$ & $2^{202}$ & $2^{154}(\overrightarrow{30})$ & $\mathrm{N} / \mathrm{A}$ & $2^{229}(5|5| 6)$ \\
\hline BLAKE-256 & HAIFA & 256 & $2^{55}$ & $\mathrm{~N} / \mathrm{A}$ & $2^{213}$ & $\mathrm{~N} / \mathrm{A}$ & $\mathrm{N} / \mathrm{A}$ \\
\hline BLAKE-512 & HAIFA & 512 & $2^{118}$ & $\mathrm{~N} / \mathrm{A}$ & $2^{419}$ & $\mathrm{~N} / \mathrm{A}$ & $\mathrm{N} / \mathrm{A}$ \\
\hline \multirow[t]{3}{*}{ Skein-512 } & HAIFA & 512 & $2^{90}$ & $\mathrm{~N} / \mathrm{A}$ & $2^{419}$ & $\mathrm{~N} / \mathrm{A}$ & $\mathrm{N} / \mathrm{A}$ \\
\hline & & & & & & \multicolumn{2}{|c|}{ Key recovery } \\
\hline & & & & & & 16 & New 6 ] \\
\hline Streebog & $\mathrm{HAIFA}+\sigma$ & 512 & $\infty$ & $\mathrm{N} / \mathrm{A}$ & $2^{419}$ (4) & $\mathrm{N} / \mathrm{A}$ & $2^{419}$ \\
\hline
\end{tabular}

\subsection{Framework of the Attacks}

In order to recover an internal state, computed by the MAC oracle during the processing of some message, we use a framework which is similar to the framework of 16. Namely, we match states that are computed offline with (unknown) states that are computed online (during the processing of messages by the MAC oracle). However, as arbitrary states match with low probability (which does not lead to efficient attacks), we only match special states, which have a higher probability to be equal. These special states are the result of iterating random functions using chains, computed by applying the compression function on a fixed message from arbitrary initial states. In this paper, we exploit special states of two types, which were also exploited in [16]: states on which two evaluated chains collide, and states on which a single chain collides with itself to form a cycle. Additionally, some of our attacks (and in particular our attacks on HAIFA) use special states which are a result of the reduction of the image space that occurs when applying a fixed sequence of random functions.

As described above, after we compute special states both online and offline, we need to match them in order to recover an online state. However, since the online states are unknown, the matching cannot be performed directly, and we are forced to match the nodes indirectly using filters. A filter for a node (state) is a property that identifies it with high probability, i.e., once the filters of two nodes match, then the nodes themselves match with high probability. Since the complexity of the matching steps in a state-recovery attack depend on the complexity on building a filter for a node and testing a filter on a node, we are interested in building filters efficiently. In this paper, we use two types of filters: collision filters (which were also used in [16]) and diamond filters, which exploit the diamond structure (proposed in [13]) in order to build filters for a large set 
of nodes with reduced average complexity. Furthermore, in this paper we use a novel online construction of the diamond structure via the MAC oracle, whereas such a structure is typically computed offline. In particular, we show that despite the fact that the online diamond filter increases the complexity of building the filter, the complexity of the actual matching phase is significantly reduced, and gives improved attacks in many cases.

Outline. The paper is organized as follows: we begin with a description of HMAC in Section 2. We then describe and analyze the algorithms we use to compute special states in Section [3, and the filters we use in our attacks in Section 4. Next, we present a simple attack against HMAC with a HAIFA hash function in Section 5, and revisit the results of [16 in Section 6, presenting new trade-offs for attacks on Merkle-Damgård hash functions. In Section 7 , we give more complex attacks for shorter messages. Finally, in Section 8, we present our universal forgery attacks with short queries, and conclude in Section 9 .

\section{HMAC and Hash-Based MACs}

In this paper we study MAC algorithms based on a hash function, such as HMAC. HMAC is defined using a hash function $H$ as $\operatorname{HMAC}(K, M)=H(K \oplus$ opad $\| H(K \oplus$ ipad $\| M)$ ). More generally, we consider a class of designs defined as:

$$
x_{0}=I_{K} \quad x_{i+1}=h_{i}\left(x_{i}, m_{i}\right) \quad t=g\left(K, x_{p},|M|\right) .
$$

The message processing updates an internal state of size $\ell$, starting from a keydependant value $I_{K}$, and the output is produced with a key-dependant finalization function $g$. In particular, we note that the state update does not depend on the key. Our description covers HMAC [4, Sandwich-MAC [23] and envelopeMAC with any common hash function. The hash function can use the message length in the finalization process, which is a common practice, and the rounds function can depend on a block counter, as in the HAIFA mode. If the hash function uses the plain Merkle-Damgard mode, the round functions $h_{i}$ are all identical (this is the model of previous attacks [16 20]).

In this work, we assume that the tag length $n$ is larger than $\ell$, so that collision in the tag result from collisions in the internal state with very high probability. This greatly simplifies the description of the attacks, and does not restrict the scope of our results. Indeed from a function $\operatorname{MAC}_{1}(K, M)$ with an output of $n$ bits, we can build a function $\operatorname{MAC}_{2}(K, M)$ with a $2 n$-bit output by appending message blocks [0] and [1] to $M$, as $\operatorname{MAC}_{2}(K, M)=\operatorname{MAC}_{1}(K, M \|[0]) \| \operatorname{MAC}_{1}(K, M \|[1])$. Our attacks applied to $\mathrm{MAC}_{2}$ can immediately be turned to attacks on $\mathrm{MAC}_{1}$.

\section{Description and Analysis of Collision Search Algorithms}

In this section, we describe and analyze the collision search algorithms which are used in our state-recovery attacks in order to compute special states. We then 
analyze these algorithms and prove the conjectures of [16. Lemma 1 proves the first conjecture, while Lemma 3 proves the second conjecture. We also give further results in the full version of the paper [6].

\subsection{Collision Search Algorithms}

We use standard collision search algorithms, which evaluate chains starting from arbitrary points. Namely, a chain $\vec{x}$ starts from $x_{0}$, and is constructed iteratively by the equation $x_{i}=f_{i}\left(x_{i-1}\right)$ up to $i=2^{s}$ for a fixed value of $s \leq \ell / 2$. We consider two different types of collisions between two chains $\vec{x}$ and $\vec{y}$ : freeoffset collisions $\left(x_{i}=y_{j}\right.$ for any $i, j$, with all the $f_{i}$ 's being equal), and same-offset collisions $\left(x_{i}=y_{i}\right)$.

Free-Offset Collision Search. When searching offline for collisions in iterations of a fixed random function $f$, we evaluate $2^{t}$ chains starting from arbitrary points, and extended to length $2^{s}$ for $s \leq \ell / 2$.

Assuming that $2^{t} \cdot 2^{t+s} \leq 2^{\ell}$ (i.e., $2 t+s \leq \ell$ ), then each of the chains is not expected to collide with more than one other chain in the structure. This implies that the structure contains a total of about $2^{t+s}$ distinct points, and (according to the birthday paradox) we expect it to contain a total of $2^{c}=2^{2(t+s)-\ell}$ collisions. We can easily recover all of these collisions in $O\left(2^{t+s}\right)=O\left(2^{(c+\ell) / 2}\right)$ time by storing all the evaluated points and checking for collisions in memory.

We note that we can reduce the memory requirements of the algorithm by using the parallel collision search algorithm of van Oorschot and Wiener [18]. However, in this paper, we generally focus on time complexity and do not try to optimize the memory complexity of our attacks.

Same-Offset Collision Search. While free-offset collisions are the most general form of collisions, they cannot always be efficiently detected and exploited by our attacks. In particular, they cannot be efficiently detected in queries to the online oracle (as a collision between messages of different lengths would lead to different values after the finalization function). Furthermore, if the hash function uses the HAIFA iteration mode, it is also not clear how to exploit free-offset collisions offline, as the colliding chains do not merge after the collision (and thus we do not have any easily detectable non-random property).

In the cases above, we are forced to only use collisions that occur at the sameoffset. When computing $2^{t}$ chains of length $2^{s}$ (for $t$ not too large), a pair of chains collide at a fixed offset $i$ with probability $2^{-\ell}$, and thus a pair of chains collide with probability $2^{s-\ell}$. As we have $2^{2 t}$ pairs of chains, we expect to find about $2^{2 t+s-\ell}$ fixed-offset collisions.

Locating collisions online. Online collisions are detected by sorting and comparing the tags obtained by querying the MAC oracle with chains of a fixed length $2^{s}$. If we find two massages such that $\operatorname{MAC}(M)=\operatorname{MAC}\left(M^{\prime}\right)$, we can easily compute the message prefix that gives the (unknown) collision state, as described in [16]. Namely, if we denote by $M_{\mid i}$ the $i$-block prefix of $M$, then we find the smallest $i$ such that $\operatorname{MAC}\left(M_{\mid i}\right)=\operatorname{MAC}\left(M_{\mid i}^{\prime}\right)$ using binary search. This algorithm queries the 
MAC oracle with $O(s)$ messages of length $O\left(2^{s}\right)$, and thus the time complexity of locating a collision online is $s \cdot 2^{s}=\widetilde{O}\left(2^{s}\right)$.

\subsection{Analysis of the Collision Search Algorithms}

In this section, we provide useful lemmas regarding the collision search algorithms described above. These lemmas are used in order to estimate the collision probability of special states that are calculated by our attacks and thus to bound their complexity. Lemmas 1 and 2 can generally be considered as common knowledge in the field, and their proofs are given in the full version of this paper 6]. Perhaps, the most interesting results in this section are lemmas 3 and 4. These lemmas show that the probability that our collision search algorithms reach the same collision twice from different arbitrary starting points, is perhaps higher than one would expect. This phenomenon was already observed in previous works such as [18, but to the best of our knowledge, this is the first time that this lemma is formally proven. As the proof of lemma 4 is very similar to that of lemma 3, it is given in the full version of this paper [6].

Lemma 1. Let $s \leq \ell / 2$ be a non-negative integer. Let $f_{1}, f_{2}, \ldots, f_{2^{s}}$ be a sequence of random functions over the set of $2^{\ell}$ elements, and $g_{i} \triangleq f_{i} \circ \ldots \circ f_{2} \circ f_{1}$ (with the $f_{i}$ being either all identical, or independently distributed). Then, the images of two arbitrary inputs to $g_{2^{s}}$ collide with probability of about $2^{s-\ell}$, i.e. $\operatorname{Pr}_{x, y}\left[g_{2^{s}}(x)=g_{2^{s}}(y)\right]=\Theta\left(2^{s-\ell}\right)$.

Lemma 2. Let $f_{1}, f_{2}, \ldots, f_{2^{s}}$ be a sequence of random functions, then the image of the function $g_{2^{s}} \triangleq f_{2^{s}} \circ \ldots \circ f_{2} \circ f_{1}$ contains at most $\tilde{O}\left(2^{\ell-s}\right)$ points.

Lemma 3. Let $\hat{x}$ and $\hat{y}$ be two random collisions (same-offset or free-offset) found by a collision search algorithm using chains of length $2^{s}$, with a fixed $\boldsymbol{\ell}$-bit function $\boldsymbol{f}$ such that $s<\ell / 2$. Then $\operatorname{Pr}[\hat{x}=\hat{y}]=\Theta\left(2^{2 s-\ell}\right)$.

Proof. First, we note that we generally have 4 cases to analyze, according to whether $\hat{x}$ and $\hat{y}$ were found using a free-offset, or a same-offset collision search algorithm. However, the number of cases can be easily reduced to 3 , as we have 2 symmetric cases, where one collision is free-offset, and the other is same-offset. In this proof, we assume that $\hat{x}$ is a same-offset collision and $\hat{y}$ is a free-offset collision (this is the configuration used in our attacks). However, the proof can easily be adapted to the 2 other different settings.

We denote the starting points of the chains which collide on $\hat{x}$ by $\left(x_{0}, x_{0}^{\prime}\right)$, and the actual corresponding colliding points of the chains by $\left(x_{i}, x_{i}^{\prime}\right)$, and thus $f\left(x_{i}\right)=f\left(x_{i}^{\prime}\right)=\hat{x}$. In the following, we assume that $0.25 \cdot 2^{s} \leq i \leq 0.75 \cdot 2^{s}$, which occurs with probability about $1 / 2$ since the offset of the collision $\hat{x}$ is roughly uniformly distributed in the interval $\left[0,2^{s}\right] 1$ This can be shown using Lemma 1 , as increasing the length of the chains, increases the collision probability by the same multiplicative factor.

\footnotetext{
${ }^{1}$ The assumption simplifies the proof of the lower bound on the collision probability.
} 
Fixing $\left(x_{0}, x_{0}^{\prime}\right)$, we now calculate the probability that 2 chains of length $2^{s}$, starting from arbitrary points $\left(y_{0}, y_{0}^{\prime}\right)$, collide on $\hat{x}$. This occurs if $y_{0}, y_{1}, \ldots, y_{2^{s}-i}$ collides with $x_{0}, x_{1}, \ldots, x_{i}$, and $y_{0}^{\prime}, y_{1}^{\prime}, \ldots, y_{2^{s}-i}^{\prime}$ collides with $x_{0}^{\prime}, x_{1}^{\prime}, \ldots, x_{i}^{\prime}$ (or vise-versa), which happens with probability $\Theta\left(2^{2(2 s-\ell)}\right.$ ) (assuming $0.25 \cdot 2^{s} \leq$ $i \leq 0.75 \cdot 2^{s}$, all chains are of length $\left.\Theta\left(2^{s}\right)\right)$. This lower bounds the collision probability on $\hat{x}$ by $\Omega\left(2^{2(2 s-\ell)}\right)$. At the same time, the collision on $\hat{x}$ is also upper bounded by $O\left(2^{2(2 s-\ell)}\right)$, as all 4 chains are of length $O\left(2^{s}\right)$. We conclude that the collision probability on $\hat{x}$ is $\Theta\left(2^{2(2 s-\ell)}\right)$.

On the other hand, the probability that the chains starting from $\left(y_{0}, y_{0}^{\prime}\right)$ collide on any point is $\Theta\left(2^{2 s-\ell}\right)$. Assuming that the collision search algorithm evaluates $2^{t}$ chains such that $2 t+s \leq \ell$, then each evaluated chain is not expected to collide with more than one different chain, and the pairs of chains can essentially be analyzed independently.

We denote by $A$ the event that the chains starting from $\left(y_{0}, y_{0}^{\prime}\right)$ collide on $\hat{x}$, and by $B$ the event that the chains starting from $\left(y_{0}, y_{0}^{\prime}\right)$ collide. We are interested in calculating the conditional probability $\operatorname{Pr}[A \mid B]$, and we have $\operatorname{Pr}[A \mid B]=$ $\operatorname{Pr}[A \cap B] / \operatorname{Pr}[B]=\operatorname{Pr}[A] / \operatorname{Pr}[B]=\Theta\left(2^{2(2 s-\ell)-(2 s-\ell)}\right)=\Theta\left(2^{2 s-\ell}\right)$, as required.

Lemma 4. Let $\hat{x}$ and $\hat{y}$ be two arbitrary same-offset collisions found, respectively, at offsets $i$ and $j$ by a collision search algorithm using chains of fixed length $2^{s}$, with independent $\boldsymbol{\ell}$-bit functions $\boldsymbol{f}_{\boldsymbol{i}}$, such that $s<\ell / 2$. Then $\operatorname{Pr}[(\hat{x}, i)=(\hat{y}, j)]=\Theta\left(2^{s-\ell}\right)$. Furthermore, given that $i=j$, we have $\operatorname{Pr}[\hat{x}=\hat{y}]=$ $\Theta\left(2^{2 s-\ell}\right)$.

\section{$4 \quad$ Filters}

We describe the two types of filters that we use in our attacks in order to match (known) states computed offline with unknown states computed online.

\subsection{Collision Filters}

A simple filter that we use in some of our attacks was also used in the previous work of $\left[16\right.$. We build a collision filter $\left([b],\left[b^{\prime}\right]\right)$ for a state $x$ offline by finding message blocks $\left([b],\left[b^{\prime}\right]\right)$ such that the states, obtained after processing these blocks from $x$, collide. In order to build this filter, we find a collision in the underlying hash function by evaluating its compression function for about $2^{\ell / 2}$ different messages blocks from the state $x$. In order to test this filter online on the unknown node $x^{\prime}$ obtained after processing a message $m^{\prime}$, we simply check whether the tags of $m^{\prime} \|[b]$ and $m^{\prime} \|\left[b^{\prime}\right]$ collide. As the tags of $m^{\prime} \|[b]$ and $m^{\prime} \|\left[b^{\prime}\right]$ collide with probability $2^{-n}<2^{-\ell}$ if the state obtained after processing $m^{\prime}$ is not $x$, we can conclude that the collision filter identifies the state $x$ with high probability.

The complexity of building a collision filter offline is $O\left(2^{\ell / 2}\right)$. Testing the filter online requires querying the MAC oracle with $m^{\prime} \|[b]$ and $m^{\prime} \|\left[b^{\prime}\right]$, and assuming that the length of $m^{\prime}$ is $2^{s^{\prime}}$, then it requires $O\left(2^{s^{\prime}}\right)$ time. 


\subsection{Diamond Filters}

In order to build filters for $2^{t}$ nodes, we can build a collision filter for each one of them separately, requiring a total of $O\left(2^{t+\ell / 2}\right)$ time. However, this process can be optimized using the diamond structure, introduced by Kelsey and Kohno in the herding attack [13. We now recall the details of this construction.

The diamond structure is built from a set of $2^{t}$ states $x_{i}$, constructing a set of messages $m_{i}$ of length $O(t)$, such that iterating the compression function from any state $x_{i}$ using message $m_{i}$ leads to a fixed final state $y$. The structure is built in $O(t)$ iterations, where each iteration processes a layer of nodes, and outputs a smaller layer to be processed by the next iteration. This process terminates once the layer contains only one node, which is denoted by $y$.

Starting from the first layer with $2^{t}$ points, we evaluate the compression function from each point $x_{i}$ with about $2^{(\ell-t) / 2}$ random message blocks. This gives a total of about $2^{(\ell+t) / 2}$ random values, and we expect them to contain about $2^{t}$ collisions. Each collision allows to match two different values $x_{i}, x_{j}$ and to send them to a common value in the next layer, such that its size is reduced to about $1 / 2$. The message $m_{i}$ for a state $x_{i}$ is constructed by concatenating the $O(t)$ message blocks on its path leading to $y$. According to the detailed analysis of [15, the time complexity of building the structure is is $\Theta\left(2^{(\ell+t) / 2}\right)$.

Once we finish building the diamond structure, we construct a standard collision filter for the final node $y$, using some message blocks $\left([b],\left[b^{\prime}\right]\right)$. Thus, building a diamond filter offline for $2^{t}$ states requires $O\left(2^{(\ell+t) / 2}\right)$ time, which is faster than the $O\left(2^{t+\ell / 2}\right)$ time required to build a collision filter for each node separately.

In order to test the filter for a state $x_{i}$ (in the first layer of the diamond structure), on the unknown node $x^{\prime}$ obtained after processing a message $m^{\prime}$ online, we simply check whether the tags of $m^{\prime}\left\|m_{i}\right\|[b]$ and $m^{\prime}\left\|m_{i}\right\| \mid\left[b^{\prime}\right]$ collide. Assuming that the length of $m^{\prime}$ is $2^{s^{\prime}}$, then the online test requires $O\left(t+2^{s^{\prime}}\right)$ time.

Online Diamond Filter. A novel observation that we use in this paper, is that in some attacks it is more efficient to build the diamond structure online by calling the MAC oracle. Namely, we construct a diamond structure for the set of $2^{t}$ states $x_{i}$, where (the unknown) $x_{i}$ is a result of querying the MAC oracle with a message $M_{i}$. Note the online construction is indeed possible, as the construction algorithm does not explicitly require the value of $x_{i}$, but rather builds the corresponding $m_{i}$ by testing for collisions between the states (which can be detected according to collisions in the corresponding tags). However, testing for collisions online requires that all the messages $M_{i}$, for which we build the online diamond filter, are of the same length. Assuming that the messages $M_{i}$ are of length $2^{s}$, this construction requires $O\left(2^{s+(t+\ell) / 2}\right)$ calls to the compression function.

In order to test the filter for an unknown online state $x_{i}$, on a known state $x^{\prime}$, we simply evaluate the compression function from $x^{\prime}$ on $m_{i} \|[b]$ and $m_{i} \| \mid\left[b^{\prime}\right]$, and check whether the resulting two states are equal. Thus, the offline test requires $O(t)$ time. 


\section{Internal State-Recovery for NMAC and HMAC with HAIFA}

In this section, we describe the first internal state-recovery attack applicable to HAIFA (which can also be used as a distinguishing-H attack). Our optimized attack has a complexity of $\tilde{O}\left(2^{\ell-s}\right)$ using messages of length $2^{s}$, but this only applies with $s \leq \ell / 5$; the lowest complexity we can reach is $2^{4 \ell / 5}$. We note that attacks against HAIFA can also be used to attack a Merkle-Damgård hash function; this gives more freedom in the queried messages by removing the need for long series of identical blocks as in [16].

In this attack, we fix a long sequence of random functions in order to reduce the entropy of the image states, based on Lemma 11. We then use an online diamond structure to match the states computed online with states that are compute offline. The detailed attack is as follows:

Attack 1: State-recovery attack against HMAC with HAIFA Complexity $\tilde{O}\left(2^{\ell-s}\right)$, with $s \leq \ell / 5\left(\min : 2^{4 \ell / 5}\right)$

1. (online) Fix a message $C$ of length $2^{s}$. Query the oracle with $2^{u}$ messages $M_{i}=[i] \| C$. Build an online diamond filter for the set of unknown states $X$, obtained after $M_{i}$.

2. (offline) Starting from $2^{t}$ arbitrary starting points, iterate the compression function with the fixed message $C$.

3. (offline) Test each image point $x^{\prime}$, obtained in Step 2, against each of the unknown states of $X$. If a match is found, then with high probability the state reached after the corresponding $M_{i}$ is $x^{\prime}$.
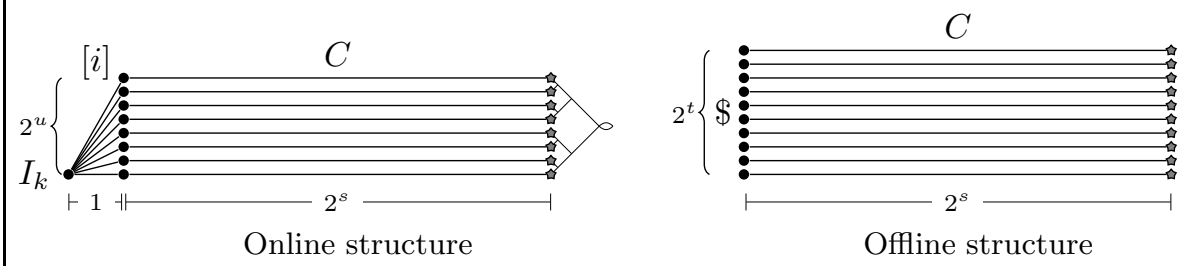

We detect a match between the grey points ( $)$ using the diamond test built online.

Complexity Analysis. In Step 3, we match the set $X$ of size $2^{u}$ (implicitly computed during Step 1), and a set of size $2^{t}$ (computed during Step 2). We compare $2^{t+u}$ pairs of points, and each pair collides with probability $2^{s-\ell}$ according to Lemma 1 Therefore, the attack is successful with high probability if $t+u \geq \ell-s$. We now assume that $t=\ell-s-u$, and evaluate the complexity of each step of the attack:

Step 1: $2^{s+u / 2+\ell / 2} \quad$ Step 2: $2^{s+t}=2^{\ell-u} \quad$ Step 3: $2^{t+u} \cdot u=2^{\ell-s} \cdot u$

The lowest complexity is reached when all the steps of the attack have the same complexity, with $s=\ell / 5$. More generally, we assume that $s \leq \ell / 5$ and we set 
$u=s$. This give an attack with complexity $\tilde{O}\left(2^{\ell-s}\right)$ since $s+u / 2+\ell / 2=$ $3 s / 2+\ell / 2 \leq 4 \ell / 5 \leq \ell-s$.

\section{New Tradeoffs for Merkle-Damgård}

In this section, we revisit the results of [16], and give more flexible tradeoffs for various message lengths.

\subsection{Trade-Off Based on Iteration Chains}

In this attack, we match special states obtained using collision, based on Lemma 3 . This attack extends the original tradeoff of 16 by using two improved techniques: first, while [16] used a fixed-offset offline collision search, we use a more general, free-offset offline collision search, which enables us to find collisions more efficiently. Second, while [16] used collision filters, we use a more efficient diamond filter.

Attack 2: Chain-based trade-off for HMAC with Merkle-Damgård Complexity $O\left(2^{\ell-s}\right)$, with $s \leq \ell / 3\left(\min : 2^{2 \ell / 3}\right)$

1. (offline) Use free-offset collision search from $2^{\ell-2 s}$ starting points with chains of length $2^{s}$, and find $2^{c}$ collisions (denoted by the set $\hat{X}$ ).

2. (offline) Build a diamond filter for the points in $\hat{X}$.

3. (online) Query the oracle with $2^{t}$ messages $M_{i}=[i] \|[0]^{2^{s}}$. Sort the tags, and locate 1 collision.

4. (online) Use a binary search to find the message prefix giving the unknown online collision state $\hat{y}$.

5. (online) Match the unknown online state $\hat{y}$ with each offline state in $\hat{X}$ using the diamond filter. If a match with $\hat{x} \in \hat{X}$ is found, then with very high probability $\hat{y}=\hat{x}$.

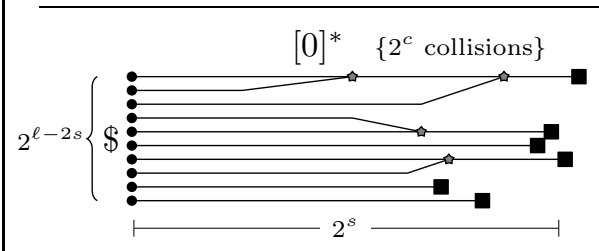

Offline structure

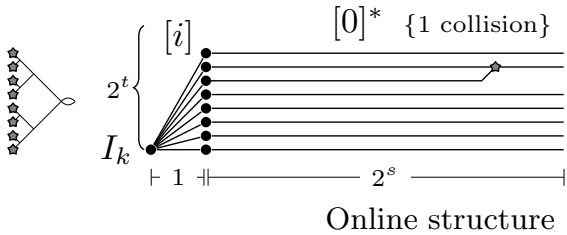

Online structure

We generate collisions offline using free-offset collision search, build a diamond filter for the collision points ( $)$, and recover the state of an online collision.

Complexity Analysis. In Step 1, we use free-offset collision search with $2^{\ell-2 s}$ starting points and chains of length $2^{s}$, and thus according to Section 3.1, we find $2^{\ell-2 s}$ collisions (i.e. $c=\ell-2 s$ ). Furthermore, according to Lemma $3 \hat{y} \in \hat{X}$ with high probability, in which case the attack succeeds. 
In Step 3, we use fixed-offset collision search with $2^{t}$ starting points and chains of length $2^{s}$, and thus according to Section 3.1. we find $2^{2 t+s-\ell}$ collisions. As we require one collision, we have $t=(\ell-s) / 2$. We now compute the complexity of each step of the attack:

$\begin{array}{rlrlrl}\text { Step 1: } & 2^{\ell / 2+c / 2} & =2^{\ell-s} & & \text { Step 2: } & 2^{\ell / 2+c / 2}=2^{\ell-s} \\ \text { Step 3: } & 2^{t+s} & =2^{(\ell+s) / 2} & & \text { Step 4: } & s \cdot 2^{s} \\ \text { Step 5: } & 2^{c+s} & =2^{(\ell+s) / 2} & & \end{array}$

With $s \leq \ell / 3$, we have $(\ell+s) / 2 \leq 2 / 3 \cdot \ell \leq \ell-s$, and the complexity of the attack is $O\left(2^{\ell-s}\right)$.

\subsection{Trade-Off Based on Cycles}

We also generalize the cycle-based state-recovery attack of [16], which uses messages of length $2^{\ell / 2}$ and has a complexity of $2^{\ell / 2}$. Our attack uses (potentially) shorter messages of length $2^{s}$ for $s \leq \ell / 2$, and has a complexity of $2^{2 \ell-3 s}$. The full attack and its analysis is given in the full version of this paper [6].

\section{Shorter Message Attacks}

In this section, we describe more complex attacks that can reach a tradeoff of $2^{\ell-2 s}$, for relatively small values of $s$. These attacks are useful in cases where the message length of the underlying hash function is very restricted (e.g. the SHA-2 family). In order to reach a complexity of $2^{\ell-2 s}$, we combine the idea of building filters in the online phase with lemmas 3 and 4 .

In the case of Merkle-Damgård with identical compression functions, we reach a complexity of $2^{\ell-2 s}$ for $s \leq \ell / 8$, i.e. the optimal complexity of this attack is $2^{3 / 4 \cdot \ell}$. With the HAIFA mode of operation, we reach a complexity of $2^{\ell-2 s}$ for $s \leq \ell / 10$ i.e. the optimal complexity of $2^{4 / 5 \cdot \ell}$, matching the optimal complexity of the attack of Section 5 .

\subsection{Merkle-Damgård}

Attack 3: Short message attack for HMAC with Merkle-Damgård Complexity $\tilde{O}\left(2^{\ell-2 s}\right)$, with $s \leq \ell / 8\left(\min : 2^{3 \ell / 4}\right)$

1. (online) Query the oracle with $2^{u}$ messages $M_{i}=[i] \|[0]^{2^{s}}$, and locate $2^{c_{1}}$ collisions.

2. (online) For each collision $(i, j)$, use a binary search to find the distance (offset) $\mu_{i j}$ from the starting point to the collision, and denote the (unknown) state reach after $M_{i}$ (or $M_{j}$ ) by $y_{i j}$.

Denote the set of all $y_{i j}$ (containing about $2^{c_{1}}$ states) by $Y$. Build an online diamond filter for all the states in $Y$. 
3. (offline) Run a free-offset collision search algorithm from $2^{t}$ starting points with chains of length $2^{s}$, and locate $2^{c_{2}}$ collisions.

4. (offline) For each offline collision $\hat{x}$, match its iterates with all points $y_{i j} \in Y$ : iterate the compression function with a zero message starting from $\hat{x}$ (up to $2^{s}$ times), and match iterate $2^{s}-\mu_{i j}$ (i.e., $f^{2^{s}-\mu_{i j}}(\hat{x})$ ) with $y_{i j}$ using the diamond filter. If a match is found, then with high probability $y_{i j}=f^{2^{s}-\mu_{i j}}(\hat{x})$.

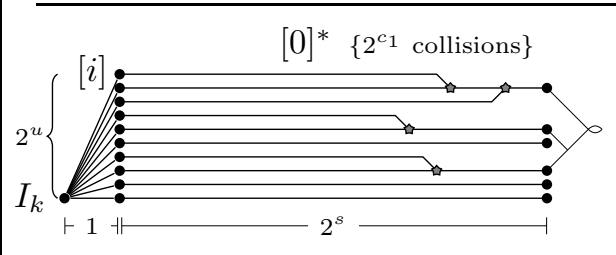

Online structure

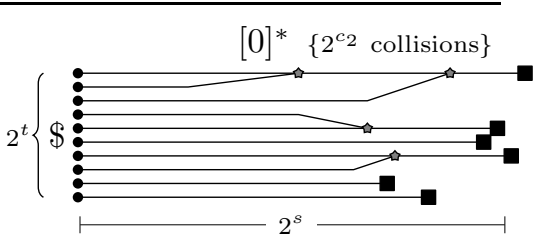

Offline structure

We generate collisions and build a diamond filter online, and match them with collisions found offline.

Complexity Analysis. Using similar analysis to Section 6.1 we have $c_{1}=$ $2 u+s-\ell$ (as a pair of chains collide at the same offset with probability $2^{s-\ell}$, and we have $2^{2 u}$ such pairs) and $c_{2}=2 t+2 s-\ell$. The attack succeeds if the sets of collisions found online and offline intersect. According to Lemma 3 this occurs with high probability if $c_{1}+c_{2} \geq \ell-2 s$. In the following, we assume $c_{1}+c_{2}=\ell-2 s$.

Step 1: $\quad 2^{u+s}=2^{s / 2+c_{1} / 2+\ell / 2}$ Step 2: $\quad 2^{s+c_{1} / 2+\ell / 2}=2^{\ell-c_{2} / 2}$

Step 3: $\quad 2^{t+s}=2^{\ell / 2+c_{2} / 2} \quad$ Step 4: $2^{c_{2}+s}+2^{c_{1}+c_{2}} \cdot c_{1}=2^{c_{2}+s}+2^{\ell-2 s} \cdot c_{1}$

The best tradeoffs are achieved by balancing steps 2 and 3 , i.e. with $c_{2}=\ell / 2$. This reduces the complexity to:

$\begin{array}{lrrr}\text { Step 1: } & 2^{3 \ell / 4-s / 2} & \text { Step 2: } & 2^{3 \ell / 4} \\ \text { Step 3: } & 2^{3 \ell / 4} & \text { Step 4: } & 2^{\ell / 2+s}+2^{\ell-2 s} \cdot \ell / 2\end{array}$

With $s \leq \ell / 8$, we have $\ell / 2+s \leq 5 \ell / 8$ and $3 \ell / 4 \leq \ell-2 s$; therefore the complexity of the attack is $\tilde{O}\left(2^{\ell-2 s}\right)$.

\subsection{HAIFA}

Since the attack is very similar to the previous attack on Merkle-Damgård, we only specify the differences between the attacks.

Attack 4: Short message attack for HMAC with HAIFA Complexity $\tilde{O}\left(2^{\ell-2 s}\right)$, with $s \leq \ell / 10\left(\min : 2^{4 \ell / 5}\right)$ 
- In Step 1 of Attack 3, we fix an arbitrary suffix $C$ of length $2^{s}$, and use $M_{i}=[i] \| C$.

- Correspondingly, in Step 3, we use a fixed-offset collision search by iterating the compression function with $C$ from $2^{t}$ starting points.

- In Step 4, we match each offline collision $\hat{x}$, only with online collisions that occur at the same offset as $\hat{x}$. Thus, for each $\hat{x}$, we test only the end point of its chain (at offset $2^{s}$ ) with the corresponding states in $Y$. Note that each $\hat{x}$ is matched with $2^{c_{1}} \cdot 2^{-s}$ states in $Y$ on average.
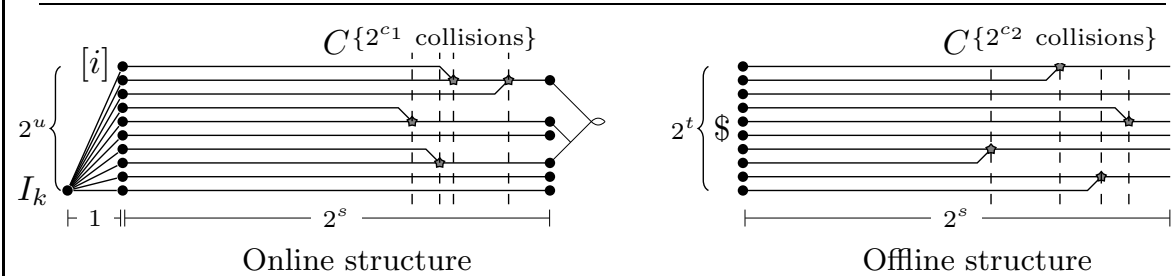

We generate collisions and build an online diamond filter, and match them with offline collisions using the collision offset as a first filter.

Analysis. The attack succeeds in case there is a match between the set of collisions detected online and offline, that occurs at the same offset. According to Lemma 4, this match occurs with high probability when $c_{1}+c_{2} \geq \ell-s$, and thus we assume that $c_{1}+c_{2}=\ell-s$.

Complexity Analysis. Similar to the analysis of the previous attacks, we have $c_{1}=2 u+s-\ell$ and $c_{2}=2 t+s-\ell$.

$$
\begin{array}{llll}
\text { Step 1: } & 2^{u+s}=2^{s / 2+c_{1} / 2+\ell / 2} & \text { Step 2: } & 2^{s+c_{1} / 2+\ell / 2}=2^{\ell-c_{2} / 2+s / 2} \\
\text { Step 3: } & 2^{s+t}=2^{s / 2+c_{2} / 2+\ell / 2} & \text { Step 4: } & 2^{c_{1}+c_{2}-s} \cdot u=2^{\ell-2 s} \cdot u
\end{array}
$$

The best tradeoffs are achieved by balancing steps 2 and 3 , i.e. with $c_{2}=\ell / 2$. This reduces the complexity to:

\section{Step 1: $2^{3 \ell / 4}$ Step 2: $2^{3 \ell / 4+s / 2}$ Step 3: $2^{3 \ell / 4+s / 2}$ Step 4: $2^{\ell-2 s} \cdot 3 \ell / 4$}

With $s \leq \ell / 10$, we have $3 \ell / 4+s / 2 \leq 4 \ell / 5 \leq \ell-2 s$; therefore the complexity of the attack is $\tilde{O}\left(2^{\ell-2 s}\right)$.

\section{Universal Forgery Attacks with Short Queries}

We now revisit the universal forgery attack of Peyrin and Wang [20. In this attack, the adversary receives a challenge message of length $2^{t}$ at the beginning of the game, and interacts with the oracle in order to predict the tag of the challenge. The attack of [20] has two phases, where in the first phase, the adversary recovers the internal state of the MAC at some step during the computation on the challenge. In the second phase, the adversary uses a second-preimage attack on 
long messages in order to generate a different message with the same tag as the challenge.

The main draw back of the attack of Peyrin and Wang (as well as its recent improvement [9]) is that its first phase uses very long queries to the MAC oracle, regardless of the length of the challenge. In this section, we use the tools developed in this paper to devise two universal forgery attacks which use shorter queries to the MAC oracle. Our first universal forgery attack has a complexity of $2^{\ell-t}$ for $t \leq \ell / 7$, using queries to the MAC oracle of length of at most $2^{2 t}$ (which is much smaller than $2^{\ell / 2}$ for any $t \leq \ell / 7$ ). Thus, the optimal complexity of this attack is $2^{6 \ell / 7}$, obtained with a challenge of length at least $2^{\ell / 7}$. Our second universal forgery attack has a complexity of only $2^{\ell-t / 2}$. However, it is applicable for any $t \leq 2 \ell / 5$, using queries to the MAC oracle of length of at most $2^{t}$. Thus, this attack has an improved optimal complexity of $2^{4 \ell / 5}$, which is obtained with a challenge of length at least $2^{2 \ell / 5}$.

In order to devise our attacks, we construct different state-recovery algorithms than the one used in [20], but reuse its second phase (i.e., the second-preimage attack) in both of the attacks. Thus, in the following, we concentrate of the staterecovery algorithms, and note that since the complexity of the second phase of the attack is $2^{\ell-t}$ for any value of $t$, it does not add a significant factor to the time complexity.

\subsection{A Universal Forgery Attack Based on the Reduction of the Image-Set Size}

Directly matching the $2^{t}$ states of the challenge message with some states evaluated offline is too expensive. Thus, we first reduce the number of nodes we match by computing and matching the images of the states under iterations of a fixed function. After matching the images, we can efficiently match and recover on the states of the challenge message.

We denote the challenge message as $C$, and the first $\kappa$ blocks of $C$ as $C_{\mid \kappa}$. The details of the first phase of the attack are as follows.

Attack 5: Universal forgery attack based on the reduction of the image-set size (first phase)

Complexity $\tilde{O}\left(2^{\ell-t}\right)$, with $t \leq \ell / 7\left(\min : 2^{6 \ell / 7}\right)$

1. (online) Build a collision filter for the last (unknown) state $z$ obtained during the computation of $\operatorname{MAC}(C)$.

2. (online) Query the oracle with $2^{t}$ messages $M_{i}=C_{\mid i} \|[0]^{2 t}-i$. Denote the set of (unknown) final states of the chains by $Y$. Build a diamond filter for all states in $Y$.

3. (offline) Compute a structure of chains containing a total of $2^{\ell-t}$ points. Each chain is extended to a maximal length of $2^{2 t+1}$, or until it collides with a previous chain. Consider the set $X$ of the $2^{2 t}$ final states of all 
the chains. According to Lemma 2 this set contains (no more than) about $2^{\ell-2 t}$ distinct points, as all the points are in the image of $f^{2^{2 t}}$.

4. (offline) Match all the points $x \in X$ with the $2^{t}$ points in $Y$. For each match between $x \in X$ and an online state in $Y$ (obtained using $M_{i}$ ), restart the chains that merge into $x$, in order to locate all the points at a (backward) distance of $2^{2 t}-i$ from $x$. Denoted this set by $\operatorname{CAND}(x)$.

5. (offline) Test the candidates: for each $x^{\prime} \in \operatorname{CAND}(x)$, compute the state obtained by following the last $2^{t}-i$ blocks of the challenge message, and match this state with $z$ using the collision filter. When a match is found, the state obtained after $C_{\mid i}$ is $x^{\prime}$ with high probability.
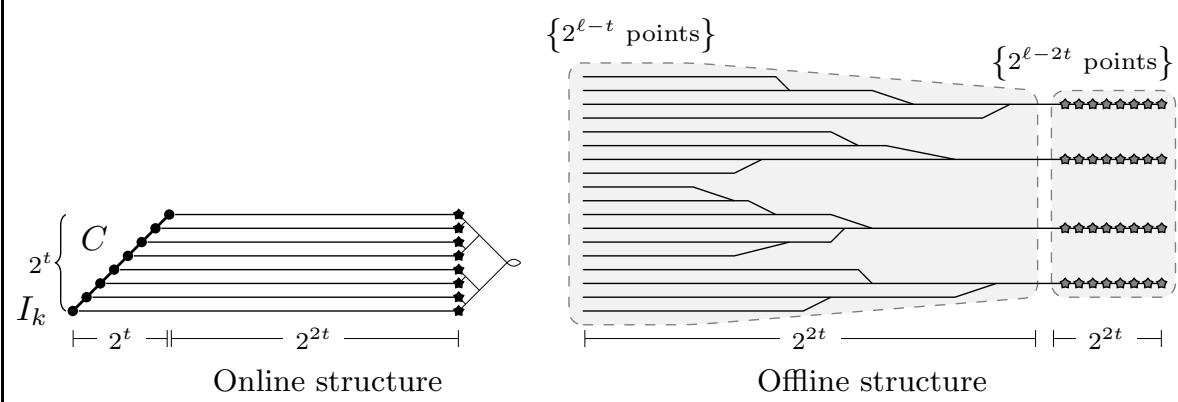

We efficiently detect a match between the challenge points $(\bullet)$ and the first part of the offline structure, by first matching $X(*)$ and $Y(\star)$.

Analysis. The structure of Step 3 contains $2^{\ell-t}$ points, and thus according to the birthday paradox, it covers one of the $2^{t}$ points of the challenge with high probability. In this case, the attack will successfully recover the state of the covered point with high probability, as we essentially have in the offline set $X$ almost all $2^{\ell-2 t}$ images of $f^{2^{2 t}}$ (including the image of the covered point).

As $X$ contains almost all $2^{\ell-2 t}$ images of $f^{2^{2 t}}$, we expect a match for every point in $Y$ in Step 3 (a total of $2^{t}$ matches). In order to calculate the expected size of $\operatorname{CAND}(x)$ in Step 5, we first calculate the expected number of the endpoints of the chains computed in Step 3 of the attack. As the chains are of length of $2^{2 t+1}$, we expect that after evaluating the first $2^{\ell-4 t}$ chains, a constant fraction of the chains will not collide with any other chain, and thus we have (at least) about $2^{\ell-4 t}$ endpoints. Since the structure contains a total of $2^{\ell-t}$ points, each endpoint is a root of a tree of average size of (at most) $2^{\ell-t-(\ell-4 t)}=2^{3 t}$. This gives about $2^{3 t-2 t}=2^{t}$ candidates nodes $\operatorname{CAND}(x)$ at a fixed (backwards) distance (note that each $x^{\prime} \in \operatorname{CAND}(x)$ is extended with a message of length about $2^{t}$, according to the challenge).

\section{Complexity.}

$\begin{array}{lrrc}\text { Step 1: } & 2^{\ell / 2+t} & \text { Step 2: } & 2^{2 t+t / 2+\ell / 2}=2^{\ell / 2+5 t / 2} \\ \text { Step 3: } & 2^{\ell-t} & \text { Step 4: } & t \cdot 2^{\ell-t} \\ \text { Step 5: } & 2^{3 t} & & \end{array}$


With $t \leq \ell / 7$, we have $\ell / 2+5 t / 2 \leq 6 \ell / 7 \leq \ell-t$; the complexity of the first phase of the universal forgery attack is $\tilde{O}\left(2^{\ell-t}\right)$, and as the second phase has a similar complexity, this is also the complexity of the full attack.

\subsection{A Universal Forgery Attack Based on Collisions}

In this attack, we devise a different algorithm which recovers one of the states computed during the execution of the challenge message. The main idea here is to find collisions between chains evaluated online, and directly match them with collisions obtained offline. This is different from the previous algorithm, which matched the endpoints of the chains, rather than nodes on which the collisions occur. Once the collisions are matched, similarly to the previous algorithm, we obtain a small set of candidate nodes, which we match with the actual challenge nodes.

Attack 6: Universal forgery attack based on collisions (first phase) Complexity $O\left(2^{\ell-t / 2}\right)$, with $t \leq 2 \ell / 5\left(\min : 2^{4 \ell / 5}\right)$

1. (online) Query the oracle with $2^{t}$ messages $M_{i}=C_{\mid i} \|[0]^{2^{t+1}}-i$, and sort the tags.

2. (online) Execute state-recovery Attack 2 using messages of length $\min \left(2^{t}, 2^{\ell / 3}\right)$, and denote by $W$ a message of length $2^{t}$ whose last computed state is recovered $a$

3. (online) Query the oracle with $2^{v}$ messages $W_{j}=W\|[j]\| 0^{2^{t}-1}$, sort the tags, and locate $2^{c}$ collisions with the tags computed using the messages $M_{i}$. For each collision of tags between $M_{i}$ and $W_{j}$, find the first collision point using binary search (note that the state of the collision is known, as the state obtained after processing $W$ is known). Store all the collision states $\hat{x}_{i j}$ in a sorted list, each one next to its distance $d_{i j}$ from $C_{\mid i}$.

4. (offline) Compute a structure of chains containing a total of $2^{\ell-c}$ points. Each chain is extended to a maximal length of $2^{t+1}$, or until it collides with a previous chain.

5. (offline) For each offline point in the structure $y$ which collides with an online collision $\hat{x}_{i j}$ (i.e., $y=\hat{x}_{i j}$ ), retrieve candidate points $\operatorname{CAND}(y)$ for the state obtained after processing $C_{\mid i}$. This is done by computing the $d_{i j}$-preimage points of $y$ in the structure (i.e., the points which are at distance $d_{i j}$ backwards from $y$ ). Assume that for each $y=\hat{x}_{i j}$, we have an average of $2^{u}$ candidate points, and thus we have a total of at most $2^{c+u}$ candidate points to test in the set $\bigcup_{i j}\left(\operatorname{CAND}\left(y=\hat{x}_{i j}\right)\right)$. Build a diamond filter for all the $2^{c+u}$ candidate points.

6. (online) For each $\left(\hat{x}_{i j}, y\right)$, match the state obtained after $C_{\mid i}$ with all the corresponding $2^{u}$ candidate points in $\operatorname{CAND}(y)$ using the diamond filter. If a match is found, then with high probability the state obtained after processing $C_{\mid i}$ is equal to the tested candidate. 


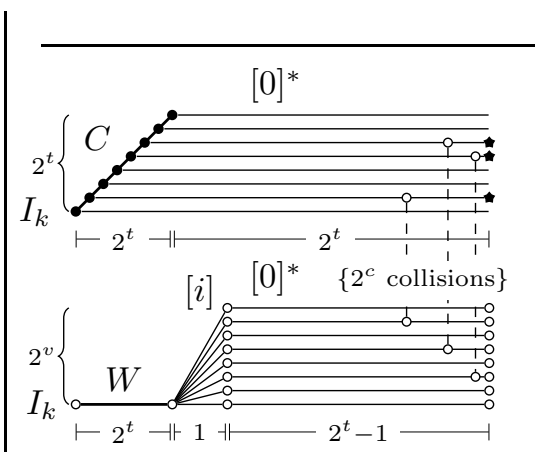

Online structure

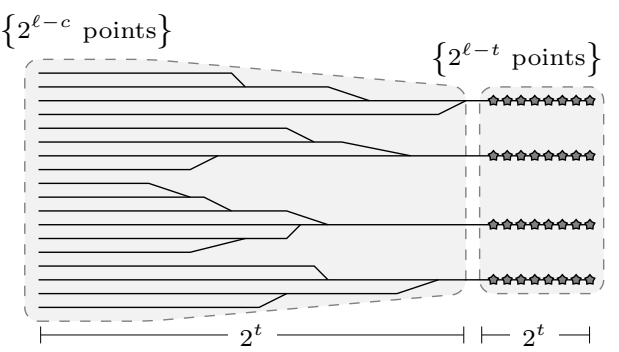

Offline structure

We match the known points in $X(*)$ and $Y($ ( ) $)$ in order to detect a match between the challenge points $(\bullet)$ and the first part of the offline structure.

\footnotetext{
${ }^{a}$ In case $t>\ell / 3$, we first recover the last computed state of a message of size $2^{\ell / 3}$, and then complement it arbitrarily to a length of $2^{t}$.
}

Analysis. In Step 3 of the attack, we find $2^{c}$ collisions between pairs of chains, where the prefix of one chain in each pair is some challenge prefix $C_{\mid i}$. Thus, the $2^{c}$ collisions cover $2^{c}$ such challenge prefixes, and moreover, the offline structure, computed in Step 4, contains $2^{\ell-c}$ points. Thus, according to the birthday paradox, with high probability, the offline structure covers one of the states obtained after the computation of a prefix $C_{\mid i}$, such that the message $M_{i}$ collides with some $W_{j}$ on a point $\hat{x}_{i j}$ in Step 3. Since the state obtained after the computation of $C_{\mid i}$ is covered by the offline structure, then $\hat{x}_{i j}$ is also covered by the offline structure, and thus the state corresponding to $C_{\mid i}$ will be matched as a candidate and recovered in Step 6.

In order to calculate the value of $c$, note that the online structure, computed in Step 1, contains $2^{t}$ chains, each of length at least $2^{t}$, and thus another arbitrary chain of length $2^{t}$ collides with one of the chains in this structure at the same offset with probability of about $2^{2 t-\ell}$. Since the structure computed in Step 3 contains $2^{v}$ such chains, the expected number of detected collisions between the structures is $2^{c}=2^{2 t+v-\ell}$, i.e., $c=2 t+v-\ell$.

In order to calculate the value of $u$, we first calculate the expected number of the endpoints of the chains computed in Step 3 of the attack. As the chains are of length of $2^{t+1}$, after evaluating the first $2^{\ell-2 t}$ chains, a constant fraction of the chains will not collide with any other chain, and thus we have (at least) about $2^{\ell-2 t}$ endpoints. Since the structure contains a total of $2^{\ell-c}$ points, each endpoint is a root of a tree of average size of (at most) $2^{\ell-c-(\ell-2 t)}=2^{2 t-c}$. This gives about $2^{2 t-c-t}=2^{t-c}$ candidates nodes at a fixed depth, i.e., $u=t-c=\ell-t-v$.

We note that the last argument we use here is heuristic, as we assume that the average number of preimages at a certain distance for the specific collision points $y$ is similar to the average for arbitrary points. However, steps 4 and 5 are not bottlenecks of the attack (as described in the complexity analysis below), and thus even if their complexity is somewhat higher, it will not effect the complexity 
of the full attack. Furthermore, we can perform a more complicated matching phase, in which we iteratively build filters for the offline structure at depths about $2^{t-1}, 2^{t-2}, \ldots$, and match them with the online structure. This guarantees that the expected complexity of the attack is as claimed below.

$\begin{array}{lcrr}\text { Step 1: } & 2^{2 t} & \text { Step 2: } & \max \left(2^{\ell-t}, 2^{2 \ell / 3}\right) \\ \text { Step 3: } & 2^{v+t} & \text { Step 4: } & 2^{\ell-c}=2^{2 \ell-2 t-v} \\ \text { Step 5: } & (c+u) \cdot 2^{(c+u+l) / 2}=t \cdot 2^{\ell / 2+t / 2} & \text { Step 6: } & 2^{c+u+t}=2^{2 t}\end{array}$

We balance steps 3 and 4 by setting $v+t=2 \ell-2 t-v$, or $v=\ell-3 t / 2$. This gives a total complexity of $O\left(2^{\ell-t / 2}\right)$ for any $t \leq 2 \ell / 5$.

\section{Conclusions and Open Problems}

In this paper, we provided improved analysis of HMAC and similar hash-based MAC constructions. More specifically, we devised the first state-recovery attacks on HMAC built using hash functions based on the HAIFA mode, and provided improved trade-offs between the message length and the complexity of staterecovery attacks for HMAC built using Merkle-Damgård hash functions. Finally, we presented the first universal forgery attacks which can be applied with short queries to the MAC oracle. Since it is widely deployed, our attacks have many applications to HMAC constructions used in practice, built using GOST, the SHA family, and other concrete hash functions.

Our results raise several interesting future work items such as devising efficient universal forgery attacks on HMAC built using hash functions based on the HAIFA mode, or proving that this mode provides resistance against such attacks. At the same time, there is also a wide gap between the complexity of the best known attacks and the security proofs for HMAC built using MerkleDamgård hash functions. For example, the best universal forgery attacks on these MACs are still significantly slower than the birthday bound, which is the security guaranteed by the proofs.

\section{References}

1. Aumasson, J.P., Henzen, L., Meier, W., Phan, R.C.W.: SHA-3 proposal BLAKE. Submission to NIST (2008/2010), http://131002.net/blake/blake.pdf

2. Aumasson, J.-P., Neves, S., Wilcox-O'Hearn, Z., Winnerlein, C.: BLAKE2: Simpler, Smaller, Fast as MD5. In: Jacobson, M., Locasto, M., Mohassel, P., Safavi-Naini, R. (eds.) ACNS 2013. LNCS, vol. 7954, pp. 119-135. Springer, Heidelberg (2013)

3. Bellare, M.: New Proofs for NMAC and HMAC: Security Without CollisionResistance. In: Dwork, C. (ed.) CRYPTO 2006. LNCS, vol. 4117, pp. 602-619. Springer, Heidelberg (2006)

4. Bellare, M., Canetti, R., Krawczyk, H.: Keying Hash Functions for Message Authentication. In: Koblitz, N. (ed.) CRYPTO 1996. LNCS, vol. 1109, pp. 1-15. Springer, Heidelberg (1996) 
5. Biham, E., Dunkelman, O.: A Framework for Iterative Hash Functions - HAIFA. IACR Cryptology ePrint Archive, Report 2007/278 (2007)

6. Dinur, I., Leurent, G.: Improved Generic Attacks Against Hash-based MACs and HAIFA. IACR Cryptology ePrint Archive, Report 2014/441 (2014)

7. Dolmatov, V., Degtyarev, A.: GOST R 34.11-2012: Hash Function. RFC 6986 (Informational) (August 2013), http://www.ietf.org/rfc/rfc6986.txt

8. Ferguson, N., Lucks, S., Schneier, B., Whiting, D., Bellare, M., Kohno, T., Callas, J., Walker, J.: The Skein hash function family. Submission to NIST (2008/2010), http://skein-hash.info

9. Guo, J., Peyrin, T., Sasaki, Y., Wang, L.: Updates on Generic Attacks against HMAC and NMAC. In: Garay, J., Gennaro, R. (eds.) CRYPTO 2014, Part I. LNCS, vol. 8616, pp. 131-148. Springer, Heidelberg (2014)

10. Guo, J., Sasaki, Y., Wang, L., Wang, M., Wen, L.: Equivalent Key Recovery Attacks against HMAC and NMAC with Whirlpool Reduced to 7 Rounds. In: FSE (2014)

11. Guo, J., Sasaki, Y., Wang, L., Wu, S.: Cryptanalysis of HMAC/NMAC-Whirlpool. In: Sako, Sarkar (eds.) [21], pp. 21-40.

12. Joux, A.: Multicollisions in Iterated Hash Functions. Application to Cascaded Constructions. In: Franklin, M. (ed.) CRYPTO 2004. LNCS, vol. 3152, pp. 306-316. Springer, Heidelberg (2004)

13. Kelsey, J., Kohno, T.: Herding Hash Functions and the Nostradamus Attack. In: Vaudenay, S. (ed.) EUROCRYPT 2006. LNCS, vol. 4004, pp. 183-200. Springer, Heidelberg (2006)

14. Kelsey, J., Schneier, B.: Second Preimages on $n$-Bit Hash Functions for Much Less than $2^{n}$ Work. In: Cramer, R. (ed.) EUROCRYPT 2005. LNCS, vol. 3494, pp. 474-490. Springer, Heidelberg (2005)

15. Kortelainen, T., Kortelainen, J.: On Diamond Structures and Trojan Message Attacks. In: Sako, Sarkar (eds.) [21], pp. 524-539

16. Leurent, G., Peyrin, T., Wang, L.: New Generic Attacks against Hash-Based MACs. In: Sako, Sarkar (eds.) [21], pp. 1-20.

17. Naito, Y., Sasaki, Y., Wang, L., Yasuda, K.: Generic State-Recovery and Forgery Attacks on ChopMD-MAC and on NMAC/HMAC. In: Sakiyama, K., Terada, M. (eds.) IWSEC 2013. LNCS, vol. 8231, pp. 83-98. Springer, Heidelberg (2013)

18. van Oorschot, P.C., Wiener, M.J.: Parallel Collision Search with Cryptanalytic Applications. J. Cryptology 12(1), 1-28 (1999)

19. Peyrin, T., Sasaki, Y., Wang, L.: Generic Related-Key Attacks for HMAC. In: Wang, X., Sako, K. (eds.) ASIACRYPT 2012. LNCS, vol. 7658, pp. 580-597. Springer, Heidelberg (2012)

20. Peyrin, T., Wang, L.: Generic Universal Forgery Attack on Iterative Hash-based MACs. In: Nguyen, P.Q., Oswald, E. (eds.) EUROCRYPT 2014. LNCS, vol. 8441, pp. 147-164. Springer, Heidelberg (2014)

21. Sako, K., Sarkar, P. (eds.): ASIACRYPT 2013, Part II. LNCS, vol. 8270. Springer, Heidelberg (2013)

22. Tsudik, G.: Message authentication with one-way hash functions. SIGCOMM Comput. Commun. Rev. 22(5), 29-38 (1992), http://doi.acm.org/10.1145/141809.141812

23. Yasuda, K.: "Sandwich" Is Indeed Secure: How to Authenticate a Message with Just One Hashing. In: Pieprzyk, J., Ghodosi, H., Dawson, E. (eds.) ACISP 2007. LNCS, vol. 4586, pp. 355-369. Springer, Heidelberg (2007) 